\title{
Antibacterial activity of leaves and flowers of Clitoria ternatea (butterfly pea plant) in Sri Lanka by using different solvent extracts
}

\author{
TD Senarathna, H Mudalige and S Dias
}

\section{Introduction}

Recent antibiotic resistant outbreaks show that it is important to discover novel natural antimicrobial agents. Clitoria ternatea is a commonly grown plant in Sri Lanka which is believed to have a high phytochemical content along with potential antibacterial activity. The current study was undertaken to determine the antibacterial activity of the leaves and flower extracted using different solvent extracts (95\% ethanol and 70\% methanol) against Escherichia coli and Staphylococcus aureus.

\section{Methodology}

Plant samples were extracted using the maceration technique and the stock solution was prepared at $100 \mathrm{mg} / \mathrm{ml}, 40 \mathrm{mg} / \mathrm{ml}$ and $20 \mathrm{mg} / \mathrm{ml}$ concentrations. Total phenolic content (TPC) was determined using Folic-Ciocalteu reagent assay while total flavonoid content (TFC) was determined based on aluminium chloride flavonoid assay. Antibacterial activity was assessed against Staphylococcus aureus (ATCC28923) and Escherichia coli (ATCC 25922) by using agar well diffusion method. Data was statistically analyzed using the IBM SPSS version 21 software.

\section{Results}

Inhibition zones against $S$. aureus in ethanolic extracts were $26.5 \pm 1.50 \mathrm{~mm}(100 \mathrm{mg} / \mathrm{ml}), 23 \pm 1.00$ $\mathrm{mm}(40 \mathrm{mg} / \mathrm{ml})$ and $7.5 \pm 0.50 \mathrm{~mm}(20 \mathrm{mg} / \mathrm{ml})$ while in methanolic extracts were $23.5 \pm 1.50 \mathrm{~mm}$ $(100 \mathrm{mg} / \mathrm{ml}), 21.6 \pm 1.00 \mathrm{~mm}(40 \mathrm{mg} / \mathrm{ml})$ and $6.5 \pm 0.40 \mathrm{~mm}(20 \mathrm{mg} / \mathrm{ml})$. Inhibition zones against E. coli in ethanolic extract were $15.50 \pm 1.50 \mathrm{~mm}(100 \mathrm{mg} / \mathrm{ml}), 13.50 \pm 1.70 \mathrm{~mm}(40 \mathrm{mg} / \mathrm{ml})$ and $6.80 \pm 1.00 \mathrm{~mm}(20 \mathrm{mg} / \mathrm{ml})$ while in methanolic extracts were $26 \pm 1.30 \mathrm{~mm}(100 \mathrm{mg} / \mathrm{ml})$, $23.10 \pm 1.00 \mathrm{~mm}(40 \mathrm{mg} / \mathrm{ml})$ and $7.5 \pm 0.50 \mathrm{~mm}(20 \mathrm{mg} / \mathrm{ml})$. A positive correlation was observed between TPC and the antibacterial activity against $S$. aureus $(\mathrm{R} \sim 0.720)$ and $E$. coli $(\mathrm{R} \sim 0.810)$ in all sample extracts separately. Similarly, positive correlation was observed between (TFC) and antibacterial activity against $S$. aureus $(\mathrm{R} \sim 0.640)$ and E. coli $(\mathrm{R} \sim 0.800)$.

\section{Conclusion}

Higher inhibition zones against $S$. aureus were observed in the ethanolic leaves and flower extracts than that of methanolic sample extracts. Higher inhibition zones against $E$. coli were observed in methanolic leaves and flower extracts than that of ethanolic sample extracts.

Keywords: Clitoria ternatea, phytochemicals, antibacterial activity.

School of Science, Business Management School (BMS).591, Galle road, Colombo 06, Sri Lanka Address for correspondence: Dr. Sajini Dias.+94768909414 Email: saj82_dias@yahoo.com iD https://orcid.org/0000-0002-8454-2733. 